\title{
Optimal H.264 Scalable Video Scheduling Policies for 3G/4G Wireless Cellular and Video Sensor Networks
}

\author{
Vamseedhar R. Reddyvari and Aditya K. Jagannatham \\ Department of Electrical Engineering, Indian Institute of Technology Kanpur, Kanpur 208016, India \\ Correspondence should be addressed to Aditya K. Jagannatham, adityaj@iitk.ac.in
}

Received 27 April 2012; Revised 27 August 2012; Accepted 19 September 2012

Academic Editor: Mei-Ling Shyu

Copyright ( $) 2012$ V. R. Reddyvari and A. K. Jagannatham. This is an open access article distributed under the Creative Commons Attribution License, which permits unrestricted use, distribution, and reproduction in any medium, provided the original work is properly cited.

\begin{abstract}
We consider the problem of optimal H.264 scalable video scheduling, with an objective of maximizing the end-user video quality while ensuring fairness in 3G/4G broadband wireless networks and video sensor networks. We propose a novel framework to characterize the video quality-based utility of the H.264 temporal and quality scalable video layers. Subsequently, we formulate the scalable video scheduling framework as a Markov decision process (MDP) for long-term average video utility maximization and derive the optimal index based-scalable video scheduling policies ISVP and ISVPF towards video quality maximization. Further, we extend this framework to multiuser and multisubchannel scenario of $4 \mathrm{G}$ wireless networks. In this context, we propose two novel schemes for long-term streaming video quality performance optimization based on maximum weight bipartite and greedy matching paradigms. Simulation results demonstrate that the proposed algorithms achieve superior end-user video experience compared to competing scheduling policies such as Proportional Fairness (PF), Linear Index Policy (LIP), Rate Starvation Age policy (RSA), and Quality Proportional Fair Policy (QPF).
\end{abstract}

\section{Introduction}

The advent of portable smart devices and broadband enabling wireless technologies such as LTE and WiMAX have led to the availability of a plethora of video applications and services such as video conferencing, multimedia streaming, interactive gaming, and real-time video monitoring in $3 \mathrm{G} /$ $4 \mathrm{G}$ wireless networks. A typical scenario in a $4 \mathrm{G}$ network is shown in the Figure 1. Video sensor networks are another paradigm which is gaining popularity due to its application in digital security and online surveillance. This demand for such wireless broadband services is expected to continue to increase in the future with progressive innovations in wireless technologies and devices leading to universal appeal of such services combined with ubiquitous availability of smart phones. Further, video content, which is the key to such popular $3 \mathrm{G} / 4 \mathrm{G}$ services, is expected to progressively comprise a dominating fraction of the wireless traffic. However, the erratic wireless environment coupled with the tremendous heterogeneity in the display and decoding capabilities of wireless devices such as smart phones, tablets, and notebooks renders conventional fixed profile video transmission unsuitable in such scenarios.

H.264 based scalable video coding (SVC) has gained significant popularity in the context of video transmission over wireless links owing to its several advantages over conventional video coding. SVC avoids the problem of simulcasting fixed profile video streams at different spatial and temporal profiles by embedding a base low resolution stream in a hierarchical stream consisting of several differential enhancement layers. Another significant advantage of SVC over conventional video coding is graceful degradation of video quality in the event of packet drops due to network congestion. As a result of these advantages, SVC is rapidly increasing in popularity as a de facto scheme for video coding in wireless networks. Reliable video transmission over bandwidth constrained wireless packet networks is further challenging compared to conventional broadband applications such as FTP and internet access due to the high delay sensitivity of video. Hence efficient video scheduling algorithms are critical towards QoS enforcement and end-user video quality maximization in broadband $4 \mathrm{G}$ networks. 


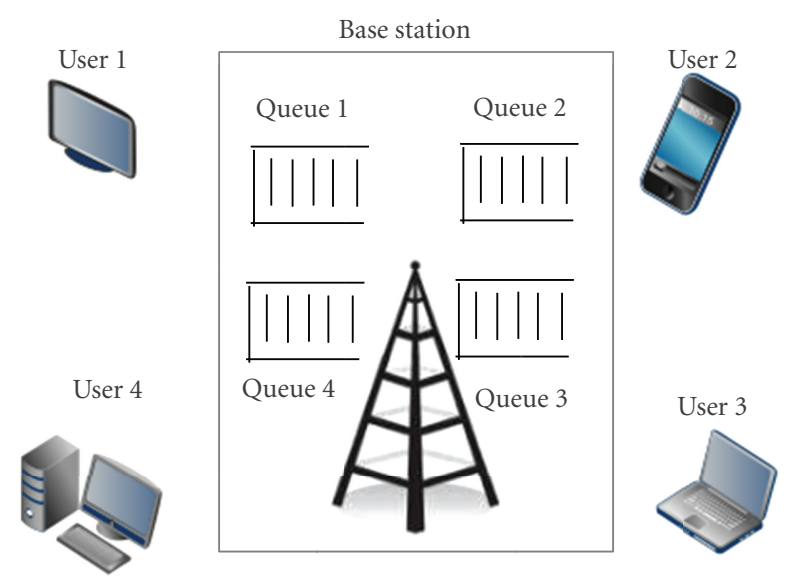

FIGURE 1: Typical 4G cellular network with heterogeneous users.

Considerable research has been carried out towards optimal resource allocation and scheduling with Quality of Service (QoS) constraints in wireless networks. The authors in [1] proposed an index-based Highest Urgency First (HUF) scheme towards multiuser scheduling which assigns an urgency parameter to each scheduling request and schedules the packets with the highest urgency factor. The authors in [2] employ distinct service classes to differentiate flows and schedule them according to priority. However, such generic data scheduling schemes are video agnostic. They do not utilize the unique structure of coded digital video and thus result in suboptimal schemes for video quality maximization. On the other front, some work has been carried out in terms of scheduling video data with maximum quality. The authors in [3] proposed a scheduling policy to maximize video quality rendered at the receiver by taking into account the temporal error concealment of the video frames at the receiver. The authors in [4] considered the problem of adaptive scheduling policy for real-time video transmission and derived optimal policies by employing a Markov decision process framework. However, both these works do not take the fairness of users into consideration while deriving the scheduling policies. Hence, in this paper, we consider the problem of optimal scheduler design for scalable video data transmission in downlink 3G/4G wireless networks with a constraint on user fairness. In this context, we present a novel framework to characterize the utility of the different scalable video layers in an H.264 SVC video stream. Further, we set up the video scheduling problem in $3 \mathrm{G}$ systems such as HSDPA where a single channel such as the high speed downlink shared channel (HS-DSCH) is scheduled amongst multiple users as an MDP and derive a novel video utility index based scalable video scheduling policy for scheduling of scalable video data in infinite queue length (ISVP) and fixed queue length (ISVPF) scenarios. Simulation results demonstrate that these schemes outperform the proportional fair resource allocation and linear index policy (LIP) based schedulers in terms of net video quality. A preliminary work on the ISVP policy has been published by us in a conference article in [5]. In this work, we have enhanced the results significantly by including multichannel multiuser scenarios and performing extensive simulations with more number of users and comparing with additional policies.

Further, OFDM/OFDMA [6, 7] has emerged as a ubiquitous technology for broadband wireless networks because of its significant advantages. In OFDM, a wideband channel can be decomposed into several parallel narrowband frequency flat wireless fading channels thus avoiding the problem of intersymbol interference in frequency selective channels. In such scenarios, efficient multiuser multi-sub-carrier channel allocation algorithms are critical towards QoS enforcement and video quality maximization in next generation networks. However as mentioned earlier, the existing scheduling algorithms $[1,2,8-10]$ are generic and do not take into account the structure of coded video transmission which results in suboptimal enduser video quality in broadband wireless access. Hence in this paper, we consider the problem of optimal multiuser 4G OFDMA channel allocation to maximize the video quality while maintaining QoS fairness amongst users.

The rest of the paper is organized as follows. In Section 2 we develop the utility framework for scalable coded videos. In Sections 3 and 4, we derive the optimal 3G video scheduling policies for infinite and finite queue sizes, respectively. In Section 6, we derive the optimality criteria for 4G OFDM channel allocation in multiuser streaming scenarios. In Section 7 we describe optimal channel allocation algorithms to achieve the above optimality criteria. In Section 8, we present the simulation results, and in Section 9, we conclude.

\section{Scalable Video Utility Framework}

H.264 supports three modes of video scalability-temporal, quality, and spatial. In our work, we consider video scheduling for temporal and quality scalable H.264 video and the extension to spatially scalable video sequences is relatively straight forward. Coded digital video streams such as H.264 employ a group of pictures (GOP) structure for differential pulse-code modulation (DPCM) based video coding. In a scalable video sequence, temporal scalability is achieved through dynamic GOP size scaling by insertion or deletion of additional temporal layers. An example of the temporally scalable GOP structure with dyadic temporal enhancement video layers is shown in Figure 2. The $T_{0}$ frames are the base layer intracoded video frames while $T_{1}$ frames are intercoded and those of subsequent layers such as $T_{2}$ are bidirectional predictively coded from frames in lower layers. Quality scalability is achieved by using different quantization parameters for the quality video layers. The base quality layer $X_{0}$ as shown in Figure 3 is coded with a coarse quantization parameter $q_{0}$. The subsequent higher layer $X_{1}$ is differentially coded with a lower quantization parameter $q_{1}$ and so on for each higher layer. The highest quality corresponds to the lowest quantization parameter $q_{\min }$. Thus, the net video rate can be scaled dynamically by appropriately choosing the temporal and quality video layers. It can be readily seen from the above GOP description that different component frames of the H.264 salable video GOP have differing 


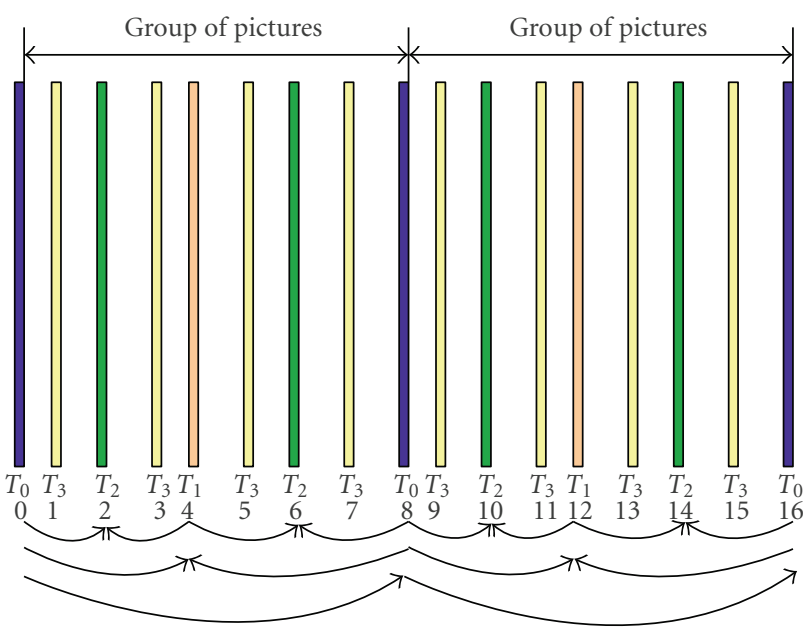

Figure 2: H.264 group of pictures (GOP) structure for temporal scalability.

impacts on the net video quality and hence have different utilities. For example considering temporal scalability, it can be observed that the base layer $T_{0}$ has a significant impact on video quality compared to the enhancement layers $T_{1}, T_{2}$, since frames in $T_{0}$ can be decoded independently as they are intracoded. However, failing reception of $T_{0}$ frames, one cannot decode the enhancement layer frames of $T_{1}, T_{2}$. Hence, a realistic video scheduling framework is needed which ascribes differentiated video utilities accurately characterizing the impact of a particular GOP component on the net video quality. Further, we define the per bit normalized utility $U_{(i, j)}$ associated with temporal layer $i$ and quality layer $j$ as the ratio of the impact on video quality $\widetilde{Q}_{(i, j)}$ to frame size $B_{(i, j)}$ as

$$
u_{(i, j)}=\frac{\widetilde{Q}_{(i, j)}}{B_{(i, j)}} .
$$

The above quantity $\boldsymbol{U}_{(i, j)}$ can be interpreted as the utility of scheduling each bit of the video layer, thus associating a higher utility with video sequences of smaller frame sizes compared to larger ones. Below, we propose a framework to compute the quality and size parameters $\widetilde{Q}_{(i, j)}, B_{(i, j)}$ in H.264 scalable video scenarios.

2.1. Video Layer Frame Size Model. The JSVM reference H.264 codec [11] developed jointly by the ITU-T H.264 and the ISO/IEC MPEG-4 AVC groups can be conveniently employed to characterize the frame sizes of the respective scalable video coded streams. Let $\mathcal{V}_{(m, n)}$ denote the scalable video stream comprising of $m+1$, that is, $0,1, \ldots, m$ temporal layers and $n+1$ quality video layers, while $\tilde{\mathcal{V}}_{(m, n)}$ denotes the exclusive $m t h$ temporal and $n$th quality layer. We consider 4 temporal layers at the standard frame rates of $3.75,7.5,15$, and 30 frames per second and 3 quantization layers in JSVM corresponding to quantization parameters (QP) 40, 36, and 32. The quantization step-size $q$ corresponding to the quantization parameter $\mathrm{QP}$ is given as $q=2^{((\mathrm{QP}-4) / 6)}[12]$.

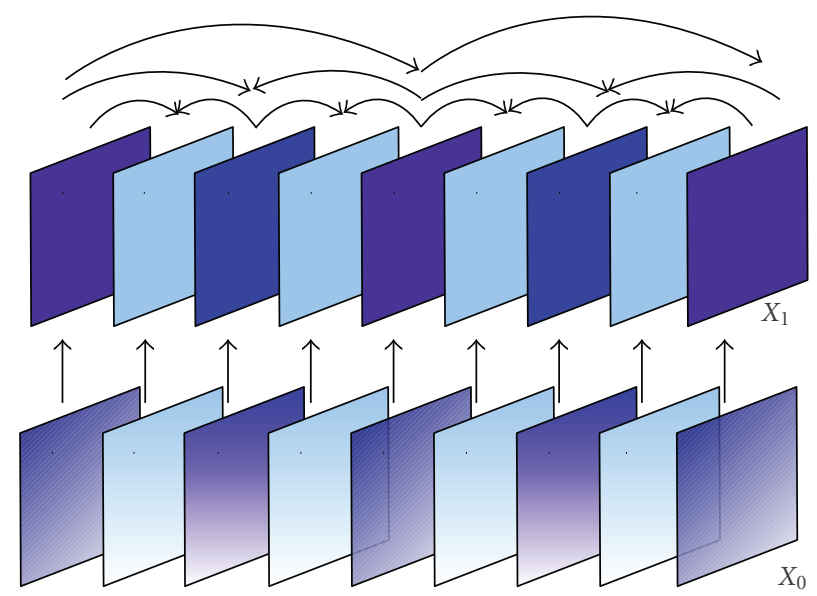

Figure 3: Temporal and quantization scalability.

Hence, the quantization step-sizes corresponding to $\mathrm{QP}=40$, 36,32 are $q=64,40.32,25.40$, respectively. We employ the notation $R_{(m, n)}$ to denote the bit-rate of the stream $\mathcal{V}_{(m, n)}$. Table 1 illustrates the computed layer rates and frame sizes for the standard Crew video [13]. For instance, the rate $R_{(0,0)}$ comprising of the spatial and quality base layers exclusively is given as $R_{(0,0)}=79.2 \mathrm{Kbps}$. Hence, the average base layer frame size can be derived by normalizing with respect to the base-layer frame rate of $f_{(0,0)}=3.75$ frames per second as

$$
B_{(0,0)}=\frac{R_{(0,0)}}{f_{(0,0)}}=21.12 \mathrm{~Kb} .
$$

The JSVM codec yields the cumulative bit-rate corresponding to the combination of base and enhancement layers of the video stream. Hence the rate $R_{(0,1)}$ corresponds to the cumulative bit-rate of the scalable video stream consisting of video layers $\tilde{\mathcal{V}}_{(0,0)}$ and $\tilde{\mathcal{V}}_{(0,1)}$. The differential rate $\widetilde{R}_{(0,1)}$ comprising exclusively of the differential video rate arising from the quality layer enhancement frames is given as

$$
\begin{aligned}
\widetilde{R}_{(0,1)} & =R_{(0,1)}-R_{(0,0)} \\
& =165.80-79.2=86.6 \mathrm{Kbps} .
\end{aligned}
$$

Further, employing the dyadic video scalability model, the exclusive rate of the $\tilde{\mathcal{V}}_{(0,1)}$ layer frames is $3.75 \mathrm{fps}$, as one such differential frame is added for each $\tilde{\mathcal{V}}_{(0,0)}$ base layer frame. Therefore, the size of each frame belonging to layer $\tilde{\mathcal{V}}_{(0,1)}$ is given as $B_{(0,1)}=86.6 / 3.75=23.09 \mathrm{~Kb}$. Similarly one can derive the differential rate and frame sizes associated with the temporal layer $\widetilde{\mathcal{V}}_{(1,0)}$. Further, as the cumulative rate $R_{(1,1)}$ incorporates the layers $\widetilde{\mathcal{V}}_{(1,1)}, \widetilde{\mathcal{V}}_{(0,1)}, \widetilde{\mathcal{V}}_{(1,0)}$, and $\widetilde{\mathcal{V}}_{(0,0)}$, the differential rate $\widetilde{R}_{(1,1)}$ is given as

$$
\widetilde{R}_{(1,1)}=\left(R_{(1,1)}-R_{(0,1)}\right)-\left(R_{(1,0)}-R_{(0,0)}\right)=32.4 \text { Kbps. }
$$

The differential bit-rates and frame sizes of the higher enhancement layers can be derived similarly. It can be noted that because of the dyadic nature of the scalability, the differential frame rates progressively double for every higher 
TABLE 1: Calculation bit rate for SVC video with 4 temporal and 3 quantization layers for Crew video.

\begin{tabular}{|c|c|c|c|c|c|c|c|}
\hline $\begin{array}{l}\text { Video } \\
\text { stream }\end{array}$ & $\begin{array}{l}\text { Cumulative rate } \\
\qquad R_{(m, n)}\end{array}$ & $\begin{array}{l}\text { Cumulative quality } \\
\qquad Q_{(m, n)}\end{array}$ & $\begin{array}{c}\text { Differential relation } \\
Y_{(m, n)}=R_{(m, n)} \text { or } Q_{(m, n)}\end{array}$ & $\begin{array}{l}\text { Differential rate } \\
\widetilde{R}_{(m, n)} \\
\end{array}$ & $\begin{array}{l}\text { Differential quality } \\
\widetilde{Q}_{(m, n)} \\
\end{array}$ & $\begin{array}{c}N \\
(\mathrm{~Kb}) \\
\end{array}$ & $\begin{array}{l}\text { Utility } \\
\boldsymbol{U}_{(m, n)}\end{array}$ \\
\hline $\mathcal{V}_{(0,0)}$ & 79.2 & 41.301 & $Y_{(0,0)}$ & 79.2000 & 41.3012 & 21.120 & 1.9556 \\
\hline $\mathcal{V}_{(0,1)}$ & 165.80 & 48.395 & $Y_{(0,1)}-Y_{(0,0)}$ & 86.6000 & 7.0943 & 23.093 & 0.3072 \\
\hline $\mathcal{V}_{(0,2)}$ & 315.80 & 53.477 & $Y_{(0,2)}-Y_{(0,1)}$ & 150.0000 & 5.0822 & 40.000 & 0.1271 \\
\hline $\mathcal{V}_{(1,0)}$ & 107.40 & 57.801 & $Y_{(1,0)}-Y_{(0,0)}$ & 28.2000 & 16.5005 & 7.520 & 2.1942 \\
\hline $\mathcal{V}_{(1,1)}$ & 226.40 & 67.730 & $\left(Y_{(1,1)}-Y_{(0,1)}\right)-\left(Y_{(1,0)}-Y_{(0,0)}\right)$ & 32.4000 & 2.8343 & 8.640 & 0.3280 \\
\hline $\mathcal{V}_{(1,2)}$ & 441.60 & 74.843 & $\left(Y_{(1,2)}-Y_{(0,2)}\right)-\left(Y_{(1,1)}-Y_{(0,1)}\right)$ & 65.2000 & 2.0304 & 17.386 & 0.1168 \\
\hline $\mathcal{V}_{(2,0)}$ & 137.50 & 67.027 & $\left(Y_{(2,0)}-Y_{(1,0)}\right) / 2$ & 15.0500 & 4.6130 & 4.013 & 1.1494 \\
\hline $\mathcal{V}_{(2,1)}$ & 292.80 & 78.541 & $\left(\left(Y_{(2,1)}-Y_{(1,1)}\right)-\left(Y_{(2,0)}-Y_{(1,0)}\right)\right) / 2$ & 18.1500 & 0.7924 & 4.840 & 0.1637 \\
\hline $\mathcal{V}_{(2,2)}$ & 575.90 & 86.788 & $\left(\left(Y_{(2,2)}-Y_{(1,2)}\right)-\left(Y_{(2,1)}-Y_{(1,1)}\right)\right) / 2$ & 33.9500 & 0.5676 & 9.053 & 0.0627 \\
\hline $\mathcal{V}_{(3,0)}$ & 171.40 & 68.735 & $\left(Y_{(3,0)}-Y_{(2,0)}\right) / 4$ & 8.4750 & 0.4269 & 2.260 & 0.1889 \\
\hline $\mathcal{V}_{(3,1)}$ & 369.70 & 80.542 & $\left(\left(Y_{(3,1)}-Y_{(2,1)}\right)-\left(Y_{(3,0)}-Y_{(2,0)}\right)\right) / 4$ & 10.7500 & 0.0733 & 2.866 & 0.0256 \\
\hline $\mathcal{V}_{(3,2)}$ & 727.30 & 89.000 & $\left(\left(Y_{(3,2)}-Y_{(2,2)}\right)-\left(Y_{(3,1)}-Y_{(2,1)}\right)\right) / 4$ & 18.6250 & 0.0525 & 4.966 & 0.0106 \\
\hline
\end{tabular}

TABLE 2: Quality parameter values $c, d$ for standard videos.

\begin{tabular}{lcccc}
\hline Video & Akiyo & City & Crew & Football \\
\hline$c$ & 0.11 & 0.13 & 0.17 & 0.08 \\
$d$ & 8.03 & 7.35 & 7.34 & 5.38 \\
\hline
\end{tabular}

enhancement layer. Hence, the frame rates associated exclusively with enhancement layers $\tilde{\mathcal{V}}_{(2,0)}$ and so forth are 7.5 and so on. Following the described procedure one can successively compute the corresponding bit-rates and associated frame sizes of the differential video layers. The bit-rates of several enhancement layers of the video sequence Crew are shown in Table 1. It can be seen that the frame sizes progressively decrease with increasing enhancement layer identifier due to the progressively increasing coding gain arising from the DPCM coding.

2.2. Video Layer Quality Model. We employ the standard video quality model proposed in $[12,14]$, which gives the quality of the scalable video stream coded at frame rate $t$ and quantization step-size $q$ as

$$
Q=Q_{\max }\left(\frac{e^{-c\left(q / q_{\min }\right)}}{e^{-c}}\right)\left(\frac{1-e^{-d\left(t / t_{\max }\right)}}{1-e^{-d}}\right),
$$

where $q_{\min }=25.40$ is the minimum quantization-step size corresponding to $\mathrm{QP}=32, t$ is frame rate or temporal resolution, $t_{\max }$ is the maximum frame rate, $Q_{\max }$ is the maximum video quality at $t=t_{\max }, q=q_{\min }$, set as $Q_{\max }=89$, and $c, d$ are the characteristic video quality parameters. The procedure for deriving the parameters $c, d$ specific to a video sequence is given in [12]. These are indicated in Table 2 for the standard video sequences Akiyo, City, Crew, and Football. The screen shots of these four standard videos are shown in Figure 4. The procedure to compute the differential video layer quality can be described as follows. Consider the standard video sequence Crew. Let the cumulative impact of the scalable video stream comprising of $m$ temporal and $n$ quality layers be denoted by $Q_{(m, n)}$. This $Q_{(m, n)}$ is calculated by substituting $m$ th layer frame rate and $n$th layer quantization parameter in (5). Hence, the quality associated with the video stream $\widetilde{\mathcal{V}}_{(0,0)}=\mathcal{V}_{(0,0)}$ consisting of the base temporal and quality layers coded at $t=3.75 \mathrm{fps}$ and $q=64$ corresponding to quantization parameter $\mathrm{QP}=40$ is given as

$$
Q_{(0,0)}=Q_{\max }\left(\frac{e^{-0.17(64 / 25.398)}}{e^{-0.17}}\right)\left(\frac{1-e^{-7.34(3.75 / 30)}}{1-e^{-7.34}}\right)=41.30 .
$$

Similarly the quality for video stream $\mathcal{V}_{(1,0)}$ with frame rate $t=7.5$ and $q=64$ is given by

$$
Q_{(1,0)}=Q_{\max }\left(\frac{e^{-0.17(64 / 25.398)}}{e^{-0.17}}\right)\left(\frac{1-e^{-7.34(7.5 / 30)}}{1-e^{-7.34}}\right)=57.80 .
$$

Employing the frame size as computed in the section above, the per-bit normalized video utility can be computed utilizing the relation in (1) as

$$
U_{(0,0)}=\frac{Q_{(0,0)}}{B_{(0,0)}}=\frac{41.30}{21.12}=1.95
$$

Thus, the above utility can be employed as a convenient handle to characterize the scheduler reward towards scheduling a particular video stream. Further, similar to the rate derivation in the above section, the quantity $Q_{(m, n)}$ denotes the cumulative quality. Hence, the differential quality $\widetilde{Q}_{(1,0)}$ associated with layer $\widetilde{\mathcal{V}}_{(1,0)}$ for instance is derived as $\widetilde{Q}_{(1,0)}=$ $Q_{(1,0)}-Q_{(0,0)}=16.50$ for Crew. The differential per-bit utility associated with layer $\tilde{\mathcal{V}}_{(1,0)}$ can be computed as, $U_{(1,0)}=2.19$ and so on. The differential layer qualities and per-bit utilities of the scalable GOP frames for the standard video sequence Crew are shown in the Table 1. The utilities of the four standard video sequences mentioned above are shown in Table 3. It can be seen from the table that the utility exhibits a decreasing trend across the enhancement layers, thus clearly demonstrating the different priorities associated with the GOP 
TABLE 3: Utility for different standard videos.

\begin{tabular}{lcccc}
\hline Video layer & Akiyo & City & Crew & Football \\
\hline$\widetilde{\mathcal{V}}_{(0,0)}$ & 12.6906 & 3.2618 & 1.9556 & 1.2266 \\
$\tilde{\mathcal{V}}_{(0,1)}$ & 1.1172 & 0.3178 & 0.3072 & 0.1185 \\
$\tilde{\mathcal{V}}_{(0,2)}$ & 0.4269 & 0.1223 & 0.1271 & 0.0466 \\
$\tilde{\mathcal{V}}_{(1,0)}$ & 24.2893 & 6.0859 & 2.1942 & 1.1310 \\
$\tilde{\mathcal{V}}_{(1,1)}$ & 2.0239 & 0.6943 & 0.3280 & 0.1049 \\
$\tilde{\mathcal{V}}_{(1,2)}$ & 0.8982 & 0.3289 & 0.1168 & 0.0389 \\
$\tilde{\mathcal{V}}_{(2,0)}$ & 9.3842 & 2.7179 & 1.1494 & 0.7410 \\
$\tilde{\mathcal{V}}_{(2,1)}$ & 0.8447 & 0.2993 & 0.1637 & 0.0639 \\
$\tilde{\mathcal{V}}_{(2,2)}$ & 0.3737 & 0.1405 & 0.0627 & 0.0223 \\
$\tilde{\mathcal{V}}_{(3,0)}$ & 1.2832 & 0.4206 & 0.1889 & 0.2299 \\
$\tilde{\mathcal{V}}_{(3,1)}$ & 0.1351 & 0.0424 & 0.0256 & 0.0170 \\
$\tilde{\mathcal{V}}_{(3,2)}$ & 0.0638 & 0.0198 & 0.0106 & 0.0063 \\
\hline
\end{tabular}

components. In the next section we derive an optimal policy towards video quality maximization while ensuring fairness in QoS.

\section{Index-Based Scalable Video Policy (ISVP)}

Employing the framework illustrated in [15], we model the scalable video scheduling scenario as a Markov decision process (MDP). The state of user $u$ at time $n$ is modeled as a combination of the channel state $s_{u}^{n}$ and the video state $v_{u}^{n}$ of the head of the queue frame of user $u$. Further, we also incorporate the user starvation age $a_{u}^{n}$ in the system state to ensure fairness in video scheduling. We assume that $s_{u}^{n} \in$ $\{1,2, \ldots L+1\}$, where each state represents a maximum bitrate $\mathcal{R}\left(s_{u}^{n}\right)$ supported by the fading channel between user $u$ and base station at time instant $n$. The vector $\mathbf{s}^{n}$ at time instant $n$ defined as $\mathbf{s}^{n}=\left[s_{1}^{n}, s_{2}^{n}, \ldots, s_{U}^{n}\right]^{T}$ characterizes the joint channel state of all users. We assume that $\left\{\mathbf{s}^{n}, n \geq\right.$ $0\}$ is an irreducible discrete time Markov Chain [16] with the $L+1$ dimensional probability transition matrix $\mathbf{P}^{u}=$ $\left[p_{i, j}^{u}\right]$. The objective of the scheduler is to allocate the shared wireless channel by scheduling the users in each time slot in such a way that maximizes the quality of scalable video without leading to starvation of users as shown in Figure 5. From the GOP structure illustrated previously in the context of scalable video, the video data state for each user $v_{u}^{n} \in$ $\{1,2, \ldots, G\}$, where $G$ is the number of frames in a GOP. Similar to above, the joint video state of the $U$ users can be denoted as $\mathbf{v}^{n}=\left[v_{1}^{n}, v_{2}^{n}, \ldots, v_{U}^{n}\right]^{T}$. The starvation age $a_{u}^{n}$ corresponds to the number of slots for which a particular user has not been served. This quantity is initialized as 0 to begin with and incremented by one for every slot for each user who is not served in that slot. If a particular user is served in the current slot, his starvation age is reset to 0 . Let $\omega(n)$ denote the user scheduled at time slot $n$. The starvation age transition for a particular user is given as

$$
a_{u}^{n}= \begin{cases}a_{u}^{n}+1, & \text { if } \omega(n) \neq u \\ 0, & \text { if } \omega(n)=u\end{cases}
$$

The total user starvation age is similarly denoted by vector $\mathbf{a}^{n}$ obtained by stacking the starvation ages of all the users. The starvation age is important parameter as it can be used to characterize the fairness. If the starvation age of all users is almost same then we can say that fairness is achieved. If the starvation age of users is low then we can say that the users are not starved. Ideally the starvation of all users should be equal and close to zero. The system state vector $\mathbf{g}=$ $\left[\left(\mathbf{v}^{n}\right)^{T},\left(\mathbf{s}^{n}\right)^{T},\left(\mathbf{a}^{n}\right)^{T}\right]^{T}$ characterizes the complete state of the system. The action $\omega(n)$ at any time instant $n$ corresponds to choosing one of the $U$ users. Employing the video utility framework developed above, the reward corresponding to serving user $u$ in slot $n$ is given as

$$
r_{n}(u)=U\left(v_{u}^{n}\right) \mathcal{R}\left(s_{u}^{n}\right)-\sum_{l \neq u} K_{l} a_{l}^{n},
$$

where $U\left(v_{u}^{n}\right)$ gives the utility of the video packet of user $u$ in state $v_{u}^{n}$ and $K_{l}$ is a constant which can control the tradeoff between quality and fairness. The transition probability from state $\mathbf{g}=\left[(\mathbf{v})^{T},(\mathbf{s})^{T},(\mathbf{a})^{T}\right]^{T}$ to $\tilde{\mathbf{g}}=\left[(\tilde{\mathbf{v}})^{T},(\tilde{\mathbf{s}})^{T},(\tilde{\mathbf{a}})^{T}\right]^{T}$ contingent on scheduling user $u$ is given as

$$
p(\tilde{\mathbf{g}} \mid \mathbf{g}, u)=p_{s_{1}, \widetilde{s}_{1}}^{1} p_{s_{2}, \widetilde{s}_{2}}^{2} \ldots p_{s_{U}, \widetilde{s}_{U}}^{U}
$$

if $\tilde{v}_{u}=v_{u}+1 \bmod G, \tilde{a}_{u}=0$ and $\tilde{a}_{z}=a_{z}+1, \tilde{v}_{z}=$ $v_{z}$ for all $z \neq u$. Our objective is to derive the optimal policy which maximizes the long-term average reward $\lim _{T \rightarrow \infty}(1 / T) E_{T}(\mathbf{g})$, where $E_{T}(\mathbf{g})$ denotes the maximum reward over $T$ time periods with initial state $\mathbf{g}$. As this is an infinite horizon problem [17] with a very large state space, conventional schemes for policy derivation are impractical. We therefore employ the novel procedure proposed in [15] to derive the optimal scalable video scheduling policy termed ISVP.

Corollary 1. An index policy $I_{u}(\mathbf{g})$ close to the optimal policy for long-term expected average reward maximization in the context of the video scheduling paradigm defined above is given as

$$
I_{u}(\mathbf{g})=u\left(v_{u}\right) \mathcal{R}\left(s_{u}\right)+K_{u} a_{u}(U+1)+K_{u} U .
$$

Proof. As described in (10), the proposed reward structure is $\mathcal{U}\left(v_{u}\right) \mathcal{R}\left(s_{u}\right)-\sum_{z \neq u} K_{z} a_{z}$. Replacing the channel state with the joint video and channel state vector $\left[\mathbf{v}^{T}, \mathbf{s}^{T}\right]^{T}$, reward with the proposed reward in (10) and applying Theorem 2 in [15] yields the desired result.

The above result guarantees that ISVP, which schedules the video user with the highest index $I_{u}(\mathbf{g})$, is close to the optimal policy and maximizes the video utility while minimizing the starvation age of all users.

\section{Finite Queue Video System Model}

The above proposed optimal ISVP policy maximizes the scalable video quality at the received users when the queue size is assumed to be infinity. In this section, we derive the optimal 


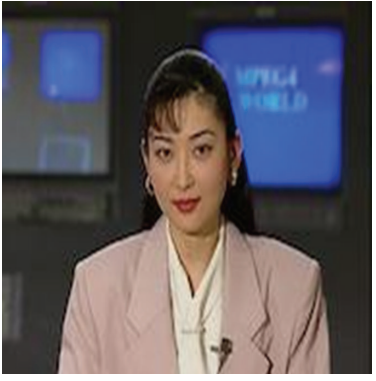

(a)

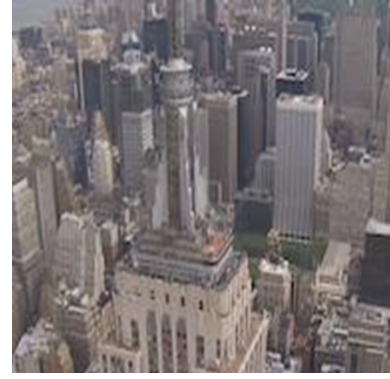

(b)

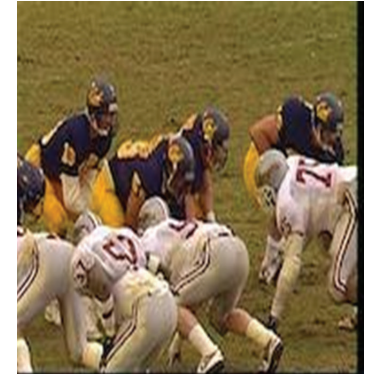

(c)

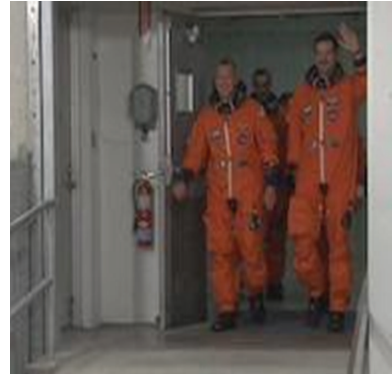

(d)

Figure 4: Screen shots of standard videos Akiyo, City, Football, and Crew.

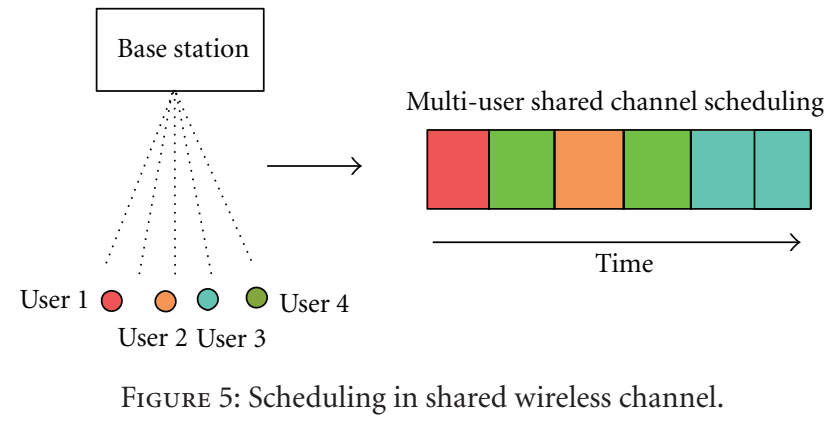

policy which maximizes scalable video quality when the buffer size in the base station is finite. The system model is identical to the one developed previously for video streaming except that the scalable video coded packets are buffered in a finite length queue at the base station. The state of user $u$ at time $n$ is modified to include the queue state $\mathbf{q}_{u}^{n}$ with the state space $\Omega_{q_{u}}$ along with the channel state $s_{u}^{n}$. The quantity $\mathbf{q}_{u}^{n}$ is a vector of utilities of the packets in the queue of user $u$ at time instant $n$. The vector $\mathbf{q}^{n}$ at time instant $n$ defined as $\mathbf{q}^{n}=\left[\mathbf{q}_{1}^{n}, \mathbf{q}_{2}^{n}, \ldots, \mathbf{q}_{U}^{n}\right]$ characterizes the queue state of all the $U$ mobile video users. Hence, the system state vector $\mathbf{g}=$ $\left[\left(\mathbf{q}^{n}\right)^{T},\left(\mathbf{s}^{n}\right)^{T},\left(\mathbf{a}^{n}\right)^{T}\right]^{T}$ characterizes the complete state of the system. The action $\omega(n)$ at any time instant $n$ corresponds to choosing one of the $U$ users. We define the reward of scheduling a user $u$ in slot $n$ as

$$
R_{u}^{n}=\mathbf{q}_{u}^{n}[1] \cdot \mathcal{R}\left(s_{u}^{n}\right)-\sum_{v \neq u} K_{v}\left(\sum_{i=1}^{i=\min \left\{a_{v}, L_{v}\right\}} \mathbf{q}_{v}^{n}[i]\right) .
$$

Observe that the reward is proportional to the rate of the channel and also utility of the scheduled video to maximize the quality of the video. Second the penalty for not scheduling a user in a time slot is proportional to the starvation age of that user. We choose this penalty function as sum of utilities of frames in the queue with number of frames as minimum of starvation age and number of frames in the queue. Hence this penalty term is implicitly proportional to the starvation age and utility of the frames of unscheduled users. The term $K_{v}$ controls the weight of penalty function compared to the positive reward. Third mathematical tractability is also considered in defining the reward. For example the chosen reward is a regenerative process which simplifies the derivation of the optimum policy in future sections.

\section{Optimal Finite Queue Length Video Scheduling Policy (ISVPF)}

Similar to the previously derived policy for infinite queue lengths, let the initial policy be a random policy meaning allocate user $u$ with probability $\alpha_{u}$. The policy iteration step is to choose the user $u$ which maximizes the index

$$
\begin{aligned}
I_{u}\left(\mathbf{i}^{n}, \mathbf{a}^{n}\right)= & \mathbf{q}_{u}^{n}[1] \cdot \mathcal{R}\left(s_{u}^{n}\right) \\
& -\sum_{v \neq u} K_{v}\left(\sum_{k=1}^{\min \left\{a_{v}^{n}, L_{v}^{n}\right\}} \mathbf{q}_{v}^{n}[k]\right) \\
& +\sum_{\mathbf{i}} P_{\mathbf{i}^{n} \mathbf{i}^{(n+1)}} h_{r}\left(\mathbf{i}^{(n+1)}, \mathbf{a}^{(n+1)}\right),
\end{aligned}
$$

where, $\mathbf{i}^{n}=\left[\mathbf{q}^{n}, \mathbf{s}^{n}\right] \in \Omega_{q_{1}} \times \cdots \times \Omega_{q_{u}} \times \Omega_{c}, \mathbf{a}^{(n+1)}=\left[a_{1}^{n}+\right.$ $\left.1, \ldots, a_{u-1}^{n}+1,0, a_{u+1}^{n}+1, \ldots, \mathbf{a}_{U}^{n}+1\right]$ and $h_{r}\left(\mathbf{i}^{(n+1)}, \mathbf{a}^{(n+1)}\right)$ is the bias term of randomized policy starting in state $\left(\mathbf{i}^{(n+1)}, \mathbf{a}^{u}\right)$. To derive the bias term, add the expression below

$$
\sum_{v} K_{v}\left(\sum_{k=1}^{\min \left\{a_{v}^{n}, L_{v}^{n}\right\}} \mathbf{q}_{v}[k]\right)-\sum_{\mathbf{i}} P_{\mathbf{i}^{n^{n}}(n+1)} h_{r}\left(\mathbf{i}^{(n+1)}, \mathbf{a}^{n}+\mathbf{e}\right)
$$

to (14), where $\mathbf{e}=[1,1, \ldots, 1]$. As this expression is independent of $u$ adding, this will not affect the outcome. The resulting equation is

$$
\begin{aligned}
I_{u}\left(\mathbf{i}^{n}, \mathbf{a}^{n}\right)= & \mathbf{q}_{u}^{n}[1] \cdot \mathcal{R}\left(s_{u}^{n}\right)+K_{u}\left(\sum_{k=1}^{\min \left\{a_{u}^{n} L_{u}^{n}\right\}} \mathbf{q}_{v}^{n}[k]\right) \\
& +\sum_{\mathbf{i}} P_{\mathbf{i}^{n} \mathbf{i}^{(n+1)}}\left(h_{r}\left(\mathbf{i}^{(n+1)}, \mathbf{a}^{u}\right)\right. \\
& \left.\quad-h_{r}\left(\mathbf{i}^{(n+1)}, \mathbf{a}^{n}+\mathbf{e}\right)\right) .
\end{aligned}
$$

To compute the index, we need to find the difference in biases. Consider two different sample paths of the stochastic process $\left(\mathbf{i}^{(n, m)}, \mathbf{a}^{(n, m)}\right), m \in\{1,2\}$ such that, $w^{1}(n)=$ $w^{2}(n), \mathbf{i}^{(n, 1)}=\mathbf{i}^{(n, 2)}, \quad n \geq 0$, and $\mathbf{a}^{(0,1)}=\mathbf{a}^{u}, \mathbf{a}^{(0,2)}=\mathbf{a}^{n}+\mathbf{e}$. 
Let $\dot{n}$ be the first time instant at which user $u$ is scheduled, then

$$
\begin{gathered}
a_{v}^{(n, 1)}=a_{v}^{(n, 2)}, \quad v \neq u, \forall n \\
a_{u}^{(n, 1)}=a_{u}^{(n, 2)}, \quad \text { if } n>\dot{n} \\
a_{u}^{(n, 1)}=n, \quad a_{u}^{(n, 2)}=a_{u}^{n}+1+n, \quad \text { if } n \leq \dot{n} .
\end{gathered}
$$

The difference in biases is equal to the average difference in the reward acquired in these two sample paths. However in this scenario, the reward differs only in cost term for user $u$ till time slot $\dot{n}=n_{0}$. Therefore,

$$
\begin{array}{r}
h_{r}\left(\mathbf{i}^{(n+1)}, \mathbf{a}^{u}\right)-h_{r}\left(\mathbf{i}^{(n+1)}, \mathbf{a}^{n}+\mathbf{e}\right) \\
=\sum_{n=1}^{n_{0}} G_{u}\left(a_{u}^{n}+n+1\right)-G_{u}(n),
\end{array}
$$

where $G_{u}^{n}(n)=K_{u}\left(\sum_{k=1}^{\min \left\{n L_{u}^{n}\right\}} \mathbf{q}_{u}^{n}[k]\right)$.

Since $n$ follows a geometrical distribution with parameter $\alpha_{u}$ the average value of the difference in biases considered above is

$$
\begin{aligned}
& r \sum_{n_{0}=1}^{\infty} \alpha_{u}\left(1-\alpha_{u}\right)^{n_{0}-1}\left(\sum_{n=1}^{n_{0}} G_{u}\left(a_{u}^{n}+n+1\right)-G_{u}(n)\right) \\
& =\alpha_{u}\left(G_{u}\left(a_{u}^{n}+2\right)-G_{u}(1)\right) \\
& +\alpha_{u}\left(1-\alpha_{u}\right)\left(G_{u}\left(a_{u}^{n}+2\right)-G_{u}(1)+G_{u}\left(a_{u}^{n}+3\right)-G_{u}(2)\right) \\
& +\alpha_{u}\left(1-\alpha_{u}\right)^{2}\left(G_{u}\left(a_{u}^{n}+2\right)-G_{u}(1)+G_{u}\left(a_{u}^{n}+3\right)\right. \\
& \left.-G_{u}(2)+G_{u}\left(a_{u}^{n}+4\right)-G_{u}(3)\right) \cdots \\
& =G_{u}\left(a_{u}^{n}+2\right)\left(\alpha_{u}+\alpha_{u}\left(1-\alpha_{u}\right)+\alpha_{u}\left(1-\alpha_{u}\right)^{2}+\cdots\right) \\
& -G_{u}(1)\left(\alpha_{u}+\alpha_{u}\left(1-\alpha_{u}\right)+\alpha_{u}\left(1-\alpha_{u}\right)^{2}+\cdots\right) \\
& +G_{u}\left(a_{u}^{n}+3\right)\left(\alpha_{u}\left(1-\alpha_{u}\right)+\alpha_{u}\left(1-\alpha_{u}\right)^{2}+\cdots\right) \\
& -G_{u}(2)\left(\alpha_{u}\left(1-\alpha_{u}\right)+\alpha_{u}\left(1-\alpha_{u}\right)^{2}+\cdots\right) \cdots \\
& =\sum_{n_{0}=1}^{\infty}\left(1-\alpha_{u}\right)^{n_{0}-1}\left(G_{u}\left(a_{u}^{n}+n_{0}+1\right)-G_{u}\left(n_{0}\right)\right) \\
& =\sum_{n_{0}=1}^{\infty}\left(1-\alpha_{u}\right)^{n_{0}-1} \\
& \times \underbrace{\left[K_{u}\left(\sum_{k=1}^{\min \left\{a_{u}^{n}+n_{0}+1, L_{u}^{n}\right\}} \mathbf{q}_{u}^{n}[k]\right)-K_{u}\left(\sum_{k=1}^{\min \left\{n_{0}, L_{u}^{n}\right\}} \mathbf{q}_{u}^{n}[k]\right)\right]}_{=\Phi\left(\mathbf{q}_{u}^{n}\right)} \\
& =\sum_{n_{0}=1}^{L_{u}^{n}}\left(1-\alpha_{u}\right)^{n_{0}-1} K_{u}\left(\sum_{k=n_{0}+1}^{\min \left\{a_{u}^{n}+n_{0}+1, L_{u}^{n}\right\}} \mathbf{q}_{u}^{n}[k]\right) \text {, }
\end{aligned}
$$

where the last equality follows from the fact that for $n_{0}>L_{u}^{n}$, the term $\Phi\left(\mathbf{q}_{u}^{n}\right)$ is equal to 0 . Hence the final index $I_{u}\left(\mathbf{i}^{n}, \mathbf{a}^{n}\right)$ for finite queue video scheduling is given by

$$
\begin{aligned}
I_{u}\left(\mathbf{i}^{n}, \mathbf{a}^{n}\right)= & \mathbf{q}_{u}^{n}[1] \cdot \mathcal{R}\left(s_{u}^{n}\right)+K_{u}\left(\sum_{k=1}^{\min \left\{a_{u}^{n}, L_{u}^{n}\right\}} \mathbf{q}_{v}^{n}[k]\right) \\
& +\sum_{n_{0}=1}^{L_{u}^{n}}\left(1-\alpha_{u}\right)^{n_{0}-1} K_{u}\left(\sum_{k=n_{0}+1}^{\min \left\{a_{u}^{n}+n_{0}+1, L_{u}^{n}\right\}} \mathbf{q}_{u}^{n}[k]\right) .
\end{aligned}
$$

\section{Multiuser OFDMA Video Streaming Model}

In the previous section, we have derived the optimal policy for a single shared channel scenario. In this section, we extend this to a multiple channel scenario by deriving an optimal channel allocation policy for $4 \mathrm{G}$ wireless systems. Consider a 4G OFDMA cellular base station BS streaming videos to a set of $U$ cellular users. Let the users be indexed by $u, 1 \leq u \leq U$. The H.264 scalable coded video packets of these users are buffered at the Base station in their respective individual queues of infinite queue lengths. Consider $N$ different OFDMA sub-channels to be allocated by the BS to the users for video transmission refer to Figure 6. Similar to the standard scheduling models established in literature we consider slotted time and channel allocation at every time slot. In this wireless video streaming scenario we wish to design a scheduler for multiuser sub-channel allocation towards video quality maximization while maintaining fairness amongst users. As already described above, the scalable coded video stream can be adapted to comprise varying combinations of temporal, quality, and spatial scalable layers. As described in Section 2, each video layer is naturally of varying utility with respect to its graded impact on the net video quality. These utility parameters for different frame components of the scalable GOP structure had been derived therein and are key towards resource allocation for video quality maximization. The normalized utility representing the per bit impact on net video quality can be computed efficiently employing the framework described in this work. Below we model this wireless video streaming scenario as a multidimensional Markov decision process (MDP) to derive the optimal multiuser multichannel OFDMA video streaming policy. Let the user state be modelled as a combination of the $N$ dimensional channel state vector, $\mathbf{s}_{u}^{n}=$ $\left[s^{n}(u, 1), s^{n}(u, 2), \ldots, s^{n}(u, N)\right]^{T}$, where $s^{n}(u, c)$ represents a maximum supported bit-rate of $\mathcal{R}\left(s^{n}(u, c)\right)$ between the BS and user $u$ over channel $c$ at time slot $n$, video state of the head of the line packet $v_{u}^{n}$. This video state corresponds to the identity of the frame in the scalable GOP. Hence the joint multiuser multichannel state $\mathbf{S}^{n} \in \mathbb{R}^{N \times U}$ can be obtained from the individual channel state vectors as $\boldsymbol{S}^{n}=$ $\left[\mathbf{s}_{1}^{n}, \mathbf{s}_{2}^{n}, \ldots, \mathbf{s}_{U}^{n}\right]$. Similarly the joint video state vector $\mathbf{v}^{n} \in \mathbb{R}^{U}$ of the $U$ users can be defined as $\mathbf{v}^{n}=\left[v_{1}^{n}, v_{2}^{n}, \ldots, v_{U}^{n}\right]^{T}$. Let the starvation age of user be denoted by $a_{u}^{n}$. This starvation age is initialised to 0 to begin with and is incremented by one in every time slot if the user is not scheduled, while being reinitialised to 0 once the user is scheduled. Hence the update 
relation for this starvation age parameter $a_{u}^{n}$ can be described as

$$
a_{u}^{n}= \begin{cases}a_{u}^{n}+1, & \text { if } u \notin \omega(n), \\ 0, & \text { if } u \in \omega(n),\end{cases}
$$

where $\omega(n) \subset\{1,2, \ldots, U\}$ is the set of users scheduled to transmit in time slot $n$. The action is choosing any $N$ users among $U$ users at every time slot. The net video quality reward of assigning users $u_{1}, u_{2}, \ldots, u_{N}$ in time slot $n$ to channels 1 through $N$, respectively, is given as

$$
r_{n}\left(u_{1}, \ldots, u_{N}\right)=\sum_{i=1}^{N} u\left(v_{u_{i}}^{n}\right) \mathcal{R}\left(s^{n}\left(u_{i}, i\right)\right)-\sum_{v \notin\left\{u_{1}, \ldots, u_{N}\right\}} K_{v} a_{v}^{n},
$$

where, $U\left(v_{u_{i}}^{n}\right)$ denotes the video utility achieved by scheduling user $u_{i}$ over the $i$ th channel and $K_{v}$ is the penalty factor corresponding to the starvation age. Deriving the optimal policy to maximize the above long-term reward for this multidimensional MDP yields the optimal scheduler policy for video quality maximization. However it is significantly challenging to obtain the optimal policy solution of this MDP. This arises due to the fact that the above MDP has an extremely large state space which grows exponentially with the number of users and channels. Thus it is inefficient to employ conventional policy and value iteration techniques to derive the optimal policy as they require a significantly large time for convergence. Hence in such scenarios, one can initialize with a random policy and employ a one step policy iteration to derive a policy that is sufficiently close to the optimal policy [18]. Now consider an initial random policy for individual user selection towards sub-channel allocation. The policy improvement step towards deriving the optimal policy for allocation of $N$ sub-channels towards video quality maximization is to choose the set of $N$ different users which maximizes the index $I_{\mathbf{u}}\left(\mathbf{i}^{n}, \mathbf{a}^{n}\right)$ given as

$$
\begin{aligned}
I_{\mathbf{u}}\left(\mathbf{i}^{n}, \mathbf{a}^{n}\right)= & \sum_{i=1}^{N} u\left(v_{u_{i}}^{n}\right) \mathcal{R}\left(s^{n}\left(u_{i}, i\right)\right) \\
& -\sum_{v \notin\left\{u_{1}, \ldots u_{N}\right\}} K_{v} a_{v}^{n}+\sum_{\mathbf{i}} P_{\mathbf{i}^{n} \mathbf{i}^{(n+1)}} h_{r}\left(\mathbf{i}^{(n+1)}, \mathbf{a}^{(\mathbf{n}+\mathbf{1})}\right),
\end{aligned}
$$

where $\mathbf{i}^{n}=\left(\mathbf{S}^{n}, \mathbf{v}^{n}\right)$ is the joint multiuser multichannel and video queue state at time slot $n$ and $h_{r}\left(\mathbf{j}^{(n+1)}, \mathbf{a}^{\mathbf{u}}\right)$ is the bias term of the randomized policy starting in state $\left(\mathbf{i}^{(n+1)}, \mathbf{a}^{(n+1)}\right)$. The starvation age of each user in $n+1$ time slot $\mathbf{a}^{(n+1)}$ is obtained by incrementing the previous starvation age by one if the user is not scheduled and assigning 0 if user is scheduled. This is represented by

$$
\mathbf{a}_{u_{i}}^{(n+1)}= \begin{cases}a_{u_{i}}^{n}+1, & \text { if } u_{i} \notin\left\{u_{1}, \ldots, u_{N}\right\} \\ 0, & \text { otherwise. }\end{cases}
$$

Hence the optimal video policy depends on the bias term which is derived employing the procedure below. Let $\mathbf{u}=$ $\left\{u_{1}, u_{1} \ldots, u_{N}\right\}$ be the set of users scheduled in time slot $n$.
To simplify the above equation, we add the following $\mathbf{u}$ independent term given as

$$
\sum_{v=1}^{U} K_{v} a_{v}^{n}-\sum_{\mathbf{i}^{(n+1)}} P_{\mathbf{i}^{n} \mathbf{i}^{(n+1)}} h_{r}\left(\mathbf{i}^{(n+1)}, \mathbf{a}^{n}+\mathbf{e}\right)
$$

where $\mathbf{e}=[1,1, \ldots, 1]^{T}$. Adding the above term to the (23), the resulting expression for the index $I_{\mathbf{u}}\left(\mathbf{i}^{n}, \mathbf{a}^{n}\right)$ can be simplified as

$$
\begin{aligned}
I_{\mathbf{u}}\left(\mathbf{i}^{n}, \mathbf{a}^{n}\right)= & \sum_{i=1}^{N} \mathcal{U}\left(v_{u_{i}}^{n}\right) \mathcal{R}\left(s^{n}\left(u_{i}, i\right)\right)+\sum_{i=1}^{N} K_{v_{i}} a_{v_{i}}^{n} \\
& +\sum_{\mathbf{i}^{(n+1)}} P_{\mathbf{i}^{n} \mathbf{i}^{(n+1)}}\left(h_{r}\left(\mathbf{i}^{n+1}, \mathbf{a}^{(n+1)}\right)-h_{r}\left(\mathbf{i}^{n+1}, \mathbf{a}^{n}+\mathbf{e}\right)\right) .
\end{aligned}
$$

From the above equation, it is clear that the optimal index depends only on the difference in the bias terms $\left(h_{r}\left(\mathbf{i}^{n+1}, \mathbf{a}^{(n+1)}\right)-h_{r}\left(\mathbf{i}^{n+1}, \mathbf{a}^{n}+\mathbf{e}\right)\right)$. To compute this consider two different sample paths of the stochastic process of the randomized policy $\left(\mathbf{i}^{(n, m)}, \mathbf{a}^{(n, m)}\right), m \in\{1,2\}$ such that $\omega^{1}(n)=\omega^{2}(n), \mathbf{i}^{(n, 1)}=\mathbf{i}^{(n, 2)}, n \geq 0$ and $\mathbf{a}^{(0,1)}=\mathbf{a}^{(n+1)}$, $\mathbf{a}^{(0,2)}=\mathbf{a}^{n}+\mathbf{e}$. Let $n_{i}$ be the first time instant at which user $u_{i}$ is scheduled. It can be readily observed that the difference in biases is equal to the average difference in the reward acquired in these two sample paths. However in this scenario, the rewards differ only in cost term for users $u_{i}$ till time slot $n_{i}$. Therefore,

$$
\begin{gathered}
a_{v}^{(n, 1)}=a_{v}^{(n, 2)}, \quad v \notin \mathbf{u}, \forall n \\
a_{u_{i}}^{(n, 1)}=a_{u_{i}}^{(n, 2)}, \quad u_{i} \in \mathbf{u}, \text { if } n>n_{i}, \\
a_{u_{i}}^{(n, 1)}=n, \quad a_{u_{i}}^{(n, 2)}=a_{u_{i}}^{n}+1+n, \quad u_{i} \in \mathbf{u}, \text { if } n \leq n_{i} .
\end{gathered}
$$

Hence, employing the above relations for the starvation age vector, the expression for the bias difference $h_{r}\left(\mathbf{i}^{(n+1)}\right.$, $\left.\mathbf{a}^{(n+1)}\right)-h_{r}\left(\mathbf{i}^{(n+1)}, \mathbf{a}^{n}+\mathbf{e}\right)$ can be derived as

$$
\begin{array}{r}
h_{r}\left(\mathbf{i}^{(n+1)}, \mathbf{a}^{(n+1)}\right)-h_{r}\left(\mathbf{i}^{(n+1)}, \mathbf{a}^{n}+\mathbf{e}\right) \\
\quad=\sum_{i=1}^{N} \sum_{n=1}^{n_{i}} K_{u_{i}}\left(a_{u_{i}}^{n}+n+1\right)-K_{u_{i}}(n) .
\end{array}
$$

Since the policy is random, $n_{i}$ is a random variable which follows geometric distribution with $p=N / U$. Therefore the 
expected value of the difference in bias term can be further simplified as

$$
\begin{aligned}
& h_{r}\left(\mathbf{i}^{(n+1)}, \mathbf{a}^{(n+1)}\right)-h_{r}\left(\mathbf{i}^{(n+1)}, \mathbf{a}^{n}+\mathbf{e}\right) \\
& =\sum_{i=1}^{N} \sum_{n_{i}=1}^{\infty} p(1-p)^{\left(n_{i}-1\right)} \sum_{i=1}^{n_{i}} K_{u_{i}}\left(a_{u_{i}}^{n}+n+1\right)-K_{u_{i}}(n) \\
& =\sum_{i=1}^{N} \sum_{n_{i}=1}^{\infty}(1-p)^{\left(n_{i}-1\right)} K_{u_{i}}\left(a_{u_{i}}^{n}+n_{i}+1\right)-K_{u_{i}}\left(n_{i}\right) \\
& =\sum_{i=1}^{N} K_{u_{i}}\left(a_{u_{i}}^{n}+1\right)\left(\sum_{n_{i}=1}^{\infty}(1-p)^{\left(n_{i}-1\right)}\right) \\
& =\frac{1}{p} \sum_{i=1}^{N} K_{u_{i}}\left(a_{u_{i}}^{n}+1\right)=\frac{U}{N} \sum_{i=1}^{N} K_{u_{i}}\left(a_{u_{i}}^{n}+1\right) .
\end{aligned}
$$

Substituting the above expression in (23), the resulting expression for the user video scheduler index $I_{\mathbf{u}}\left(\mathbf{i}^{n}, \mathbf{a}^{n}\right)$ can be simplified as

$$
\begin{aligned}
I_{\mathbf{u}}\left(\mathbf{i}^{n}, \mathbf{a}^{n}\right)= & \sum_{i=1}^{N} \mathcal{U}\left(v_{u_{i}}^{n}\right) \mathcal{R}\left(s^{n}\left(u_{i}, i\right)\right) \\
& +\sum_{i=1}^{N} K_{u_{i}} a_{u_{i}}^{n} \\
& +\frac{U}{N} \sum_{\mathbf{i}^{(n+1)}} P_{\mathbf{i}^{n} \mathbf{i}^{n+1}} \sum_{i=1}^{N} K_{u_{i}}\left(a_{u_{i}}^{n}+1\right) \\
= & \sum_{i=1}^{N} \mathcal{U}\left(v_{u_{i}}^{n}\right) \mathcal{R}\left(s^{n}\left(u_{i}, i\right)\right) \\
& +K_{u_{i}} a_{u_{i}}^{n}+K_{u_{i}}\left(a_{u_{i}}^{n}+1\right)\left(\frac{U}{N}\right) .
\end{aligned}
$$

Let $w(u, c)$ be defined as $w(u, c)=U\left(v_{u}^{n}\right) \mathcal{R}\left(s^{n}(u, c)\right)+K_{u} a_{u}^{n}+$ $K_{u}\left(a_{u}^{n}+1\right)(U / N)$. Then the index $I_{\mathbf{u}}\left(\mathbf{i}^{n}, \mathbf{a}^{n}\right)$ can be simplified as

$$
I_{\mathbf{u}}\left(\mathbf{i}^{n}, \mathbf{a}^{n}\right)=\sum_{i=1}^{N} w\left(u_{i}, i\right)
$$

Therefore the optimal video scheduler policy chooses $N$ users such that it maximises the sum component index $\sum_{i=1}^{N} w\left(u_{i}, i\right)$. Next we describe a fast algorithm to compute the multiuser multi-sub-channel allocation towards video quality maximization based on the above index optimization.

\section{Bipartite User Subchannel Index Maximization}

The above multiuser index maximization can be readily viewed as a maximum weight bipartite matching computation. Consider a bipartite graph $(U, C, E)$ such that the partitions contain the $U$ users and the $N$ channels as the nodes

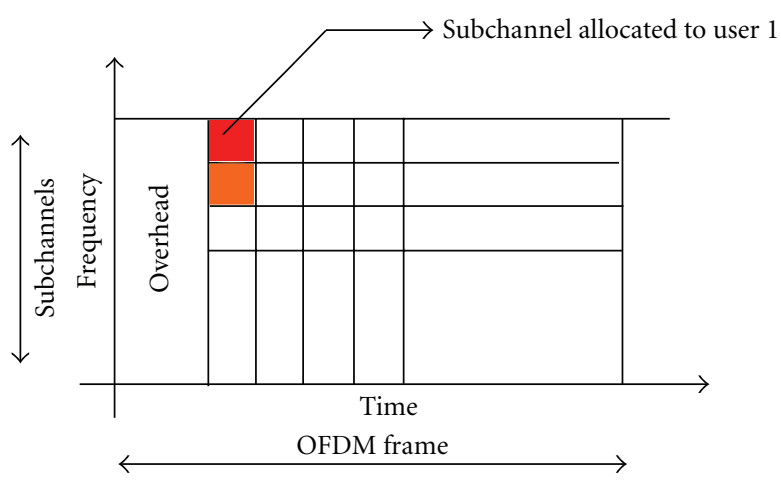

FIGURE 6: OFDM subchannel allocation with respect to time.

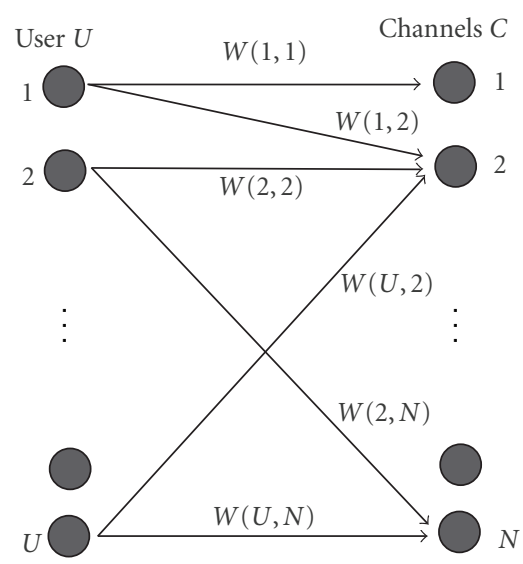

FIGURE 7: Bipartite graph with users and channels as nodes of the graph.

and the $U \times N$ edges between each user $u$ to each channel $c$ are associated with the weight $w(u, c)$ as shown in Figure 7. The optimal index computation for video scheduling thus reduces to computing the maximally wighted bipartite user sub-channel matching. This can be achieved through the Hungarian algorithm-based sub channel allocation given below. The input to the algorithm is a matrix $W$ whose $(i, j)$ th element represents weight of the edge between user $i$ and channel $j$ which is equal to $w(i, j)$. The algorithm is described below.

The Hungarian method given in Algorithm 1 thus yields the optimal index based user sub-channel allocation for long term video quality maximization. However, it has a complexity of $O\left((U+N)^{3}\right)$. Hence to reduce the computational complexity we present a suboptimal greedy algorithm of complexity $O\left(U^{2}\right)$ in Algorithm 2.

\section{Simulation Results}

We compare the performance of the proposed video optimal policies with LIP proposed in [15], proportional fair (PF) scheduling policy, and two heuristic policies. The LIP is an index policy with index $I_{u}^{l}\left(\mathbf{s}^{n}, \mathbf{a}^{n}\right)$ defined exclusively in terms of the channel state vector $\mathbf{s}^{n}$ and multiuser starvation vector $\mathbf{a}^{n}$ as $I_{u}^{l}\left(\mathbf{s}^{n}, \mathbf{a}^{n}\right)=\mathcal{R}\left(s_{u}^{n}\right)+K_{u} a_{u}(U+1)+K_{u} U$. The LIP is 


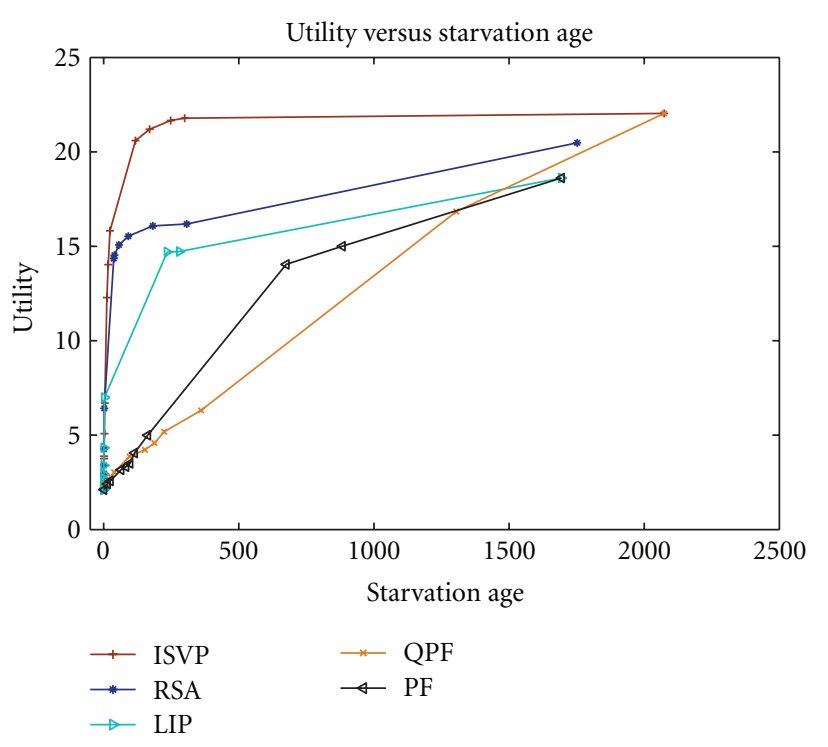

Figure 8: Utility $(\hat{\Psi})$ versus starvation age $(\hat{\chi})$.

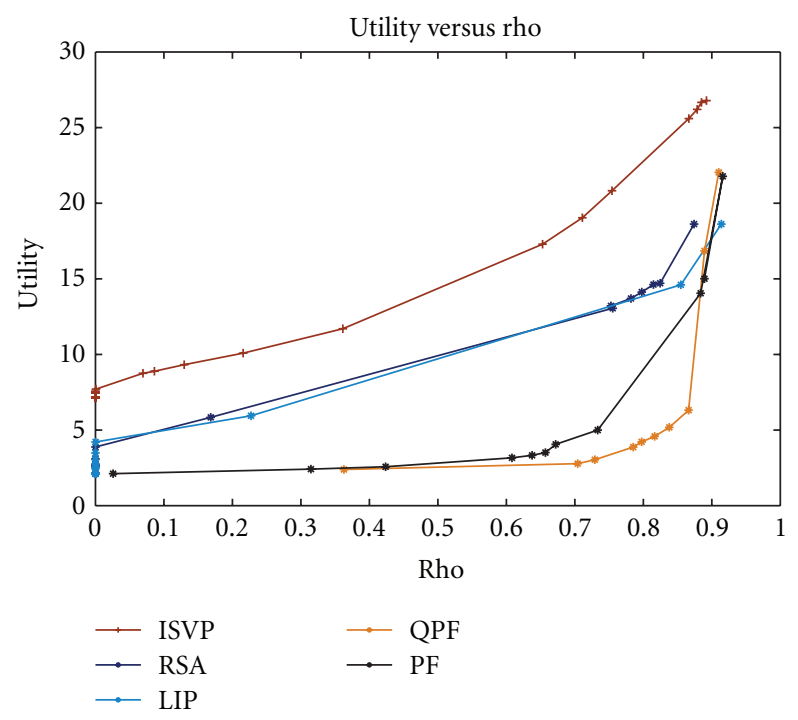

FIGURE 9: Utility $(\widehat{\Psi})$ versus Rho $\left(\widehat{\rho_{d}}\right)$.

an optimal policy for maximizing bit rate with a constraint on fairness. It is generic in sense it does not depend on the type of data transmitted. Whereas the policies ISVP, ISVPF we have proposed are suited for transmission of video data through wireless channel. These policies take advantage of the scalability of video and hence they are dynamically scalable with the state of the changing wireless environment.

The PF scheduling policy is equivalent to an index policy $I_{u}^{p}\left(\mathbf{s}^{n}\right)=\mathcal{R}\left(s_{u}^{n}\right) / Q_{u}(n)$ where $Q_{u}(n)$ is given as

$$
Q_{u}(n+1)= \begin{cases}(1-\tau) Q_{u}(n)+\tau \mathcal{R}\left(s_{u}^{n}\right), & \text { if } u=\omega(n) \\ (1-\tau) Q_{u}(n), & \text { if } u \neq \omega(n),\end{cases}
$$

where $\omega(n)$ is the scheduled user in slot $n$ and $\tau$ is the damping coefficient. As the above two policies are generic, we also compare our results with two other heuristic policies.
The first heuristic policy is the Quality Proportional Fair (QPF) policy. It is similar to PF policy except that the rate is replace by product of utility and rate. The QPF scheduling policy is equivalent to an index policy $I_{u}^{\mathrm{QPF}}\left(\mathbf{s}^{n}\right)=$ $\mathcal{U}\left(s_{u}^{n}\right) \mathcal{R}\left(s_{u}^{n}\right) / Q_{u}(n)$ where $Q_{u}(n)$ is given as

$$
Q_{u}(n+1)= \begin{cases}(1-\tau) Q_{u}(n)+\tau \mathcal{U}\left(s_{u}^{n}\right) \mathcal{R}\left(s_{u}^{n}\right), & \text { if } u=\omega(n) \\ (1-\tau) Q_{u}(n), & \text { if } u \neq \omega(n) .\end{cases}
$$

The second heuristic policy is Rate Starvation Age policy (RSA). It is an Index policy with index directly proportional to rate and starvation age. The index $I_{u}^{\mathrm{RSA}}$ is defined as $I_{u}^{\mathrm{RSA}}\left(\mathbf{s}^{n}, \mathbf{a}^{n}\right)=\mathcal{R}\left(s_{u}^{n}\right)+K_{u} a_{u}$. These policies are extended appropriately for comparison in multichannel scenarios. For multi channel scenario, we applied Greedy matching and Hungarian matching algorithms with the weights of the edges equal to above indexes $I^{\mathrm{LIP}}$ and $I^{\mathrm{PF}}$. For finite queue case whenever the buffer is full, the incoming packets are dropped.

We consider the performance measures $\Psi$, the expected per-slot long-term utility, $\chi$, the expected starvation age and $\rho_{d}$, the probability that a user is not served for longer than $d$ time slots, for evaluation of the policies. We consider an $L+$ $1=5$ channel state model with supported rate states $\mathcal{R}\left(s_{u}^{n}\right) \in$ $\{38.4,76.8,102.6,153.6,204.8\}$ Kbps. We considered $U=12$ users transmitting the standard videos Akiyo, City, Crew, and Football. We use $T=10^{5}$ slots and $P=100$ sample paths of the Markov chain. The state transition matrix is similar to the one considered in [15], with $\beta=0.999$. The $K_{u}$ value is varied for the ISVP and LIP schemes while $\tau$ is varied for the PF scheme. The starvation age and utility are calculated for different values of parameter $K_{u}$ in the range $[0,500]$. In case of the PF policy, the parameter $\tau$ is varied appropriately in the range $[0,1]$.

Figure 8 shows a comparison of the video utility of the proposed ISVP policy with LIP, PF, RSA, and QPF policies. It can be observed that the proposed ISVP policy yields the maximum video utility amongst the five competing policies. Further, as $K_{u} \rightarrow \infty$ and $\tau \rightarrow 1$, the LIP and PF policies effectively converge to the round-robin policy. Hence, the utility and starvation age coincide at this point. Further when $K_{u}=0$ the index remains same for ISVP and QPF policies so they also coincide. Figure 9 shows the plot between utility and the probability $\rho_{d}$ that a user is starved for more than $d$ slots. This is also plotted by varying the parameters as mentioned above. We observe that the utility is maximum for a particular probability for the proposed ISVP scheme compared to other policies. Thus, the proposed ISVP scheduler maximizes the net video quality while not compromising on fairness. Further the ISVP policy we proposed is an index policy and index calculation is of order $O(1)$ similar to PF and LIP policy so the cost of computation remains same which is $O(U)$.

Figures 10 and 11 show a comparison of the video utility of the proposed ISVPF policy with that LIP, RSA, QPF, and PF policies when each user queue sizes are equal to $L_{u}=500$. It can be observed that the proposed ISVPF policy yields the maximum video utility amongst the five competing policies. 
(1) Identify the largest element $\alpha$ of the matrix $W$ and replace each element $w(u, c)$ with $\alpha-w(u, c)$.

(2) From every row of the resultant matrix subtract the row minimum that is, $w(i, j)=w(i, j)-\min _{j}(w(i, j)), \forall i$.

(3) From every column of the matrix subtract the column minimum that is, $w(i, j)=w(i, j)-\min _{i}(w(i, j)) ; \forall j$.

(4) while True do

(5) In every row match a row and column if there is only one 0 in a row and strike off the other 0's in the matched column that is, $i \leftrightarrow j$, if $w(i, j)=0$, and $w\left(i^{\prime}, j\right) \neq 0, \forall i^{\prime} \neq i, \forall i \in\{1,2, \ldots, U\}$

(6) In every column match a row and column if there is only one 0 in the column and strike off the other 0's in the matched row that is, $i \leftrightarrow j$, if $w(i, j)=0$, and $w\left(i, j^{\prime}\right) \neq 0, \forall j^{\prime} \neq j, \forall j \in\{1,2, \ldots, N\}$

(7) if Allocation is not complete then

(8) Draw minimum number of lines passing through all zeroes.

(9) Identify the smallest number $\theta$ amongst all elements through which no line is passing.

(10) For each element subtract $\theta$ if no line is passing through and add $\theta$ if two lines are passing through.

(11) else

(12) break

(13) end if

(14) end while

Algorithm 1: Hungarian matching.

(1) for $i=0$ to $N$ do

(2) Match the ith channel with user $u$ having the highest weight $w(u, i)$.

(3) Set $w(u, j)=-\infty, j \neq i$.

(4) end for

Algorithm 2: Greedy matching.

Figure 9 shows the plot between utility and the probability $\rho_{d}$ that a user is starved for more than $d$ slots. We observe that the utility is maximum for a particular probability for the proposed ISVPF scheme compared to other policies. Thus, the proposed ISVPF scheduler maximizes the net video quality for finite buffers at the base station while not compromising on fairness.

In this section, we compare through simulations the performance of the proposed optimal multichannel allocation policies, Hungarian MultiChannel Optimal Allocation (HMOA), and Greedy MultiChannel Sub-Optimal Allocation (GMOA) with that of the LIP policy proposed in [15] and the standard Proportional Fair (PF) scheduler. Since these are designed for single shared channel scheduling we extend them to multichannel scheduling, by employing the Hungarian (HMLIP, HMPF) and Greedy matching (GMLIP, GMPF) paradigms described above. It can be observed from Figures 12 and 13 that the proposed HMOA policy yields the maximum video quality amongst the competing multiuser multichannel video scheduling policies. Although the GMOA is not optimal but it performs better than PF policy. Further, as $K_{u} \rightarrow \infty$ and $\tau \rightarrow 1$, the LIP and PF policies effectively converge to the round-robin policy. Hence, the utility and starvation age coincide at this point.

\section{Conclusions}

In this paper, we developed a novel framework to characterize the differential utility of the H.264 scalable video

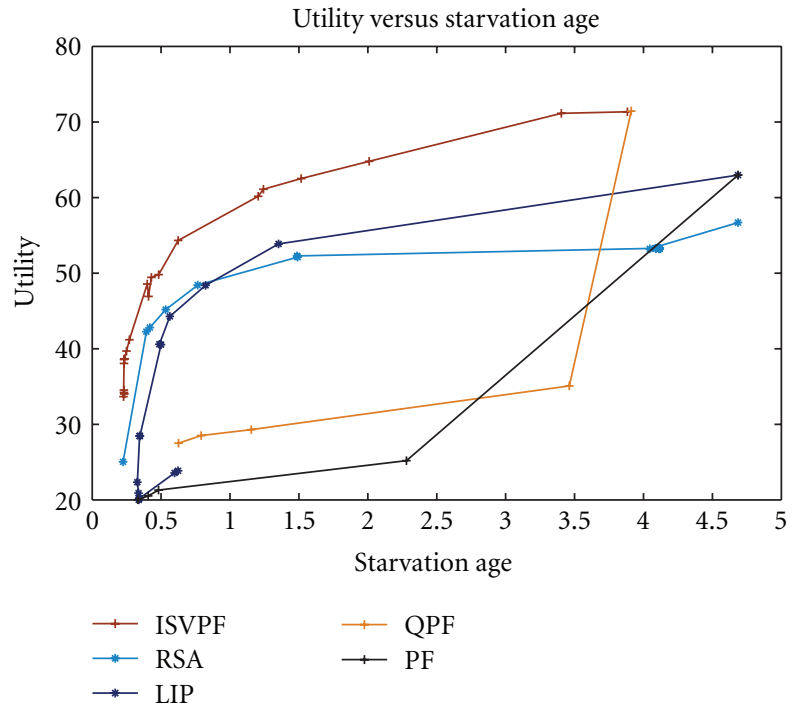

FIGURE 10: Utility $(\hat{\Psi})$ versus Starvation Age $(\hat{\chi})$ when queue length $=500$.

stream layers. Based on the proposed framework, a utilitystarvation based reward paradigm has been proposed to characterize the scheduling decisions. The end-user video quality maximization has been formulated as an appropriate Markov decision process, and an optimal index based ISVP and ISVPF have been derived towards scheduling the scalable video frames for net video quality maximization in next generation wireless networks. We demonstrated through simulations that the derived ISVP and ISVPF policies achieve better utility compared to PF, LIP, RSA, and QPF policies. Further, we extended this to multiuser multichannel scenarios. The multichannel allocation problem was formulated as an MDP, and the optimal index based channel allocation policy was derived towards video quality maximization which also ensured fairness of video QoS. Two novel index based video scheduling schemes, namely, the Hungarian and 


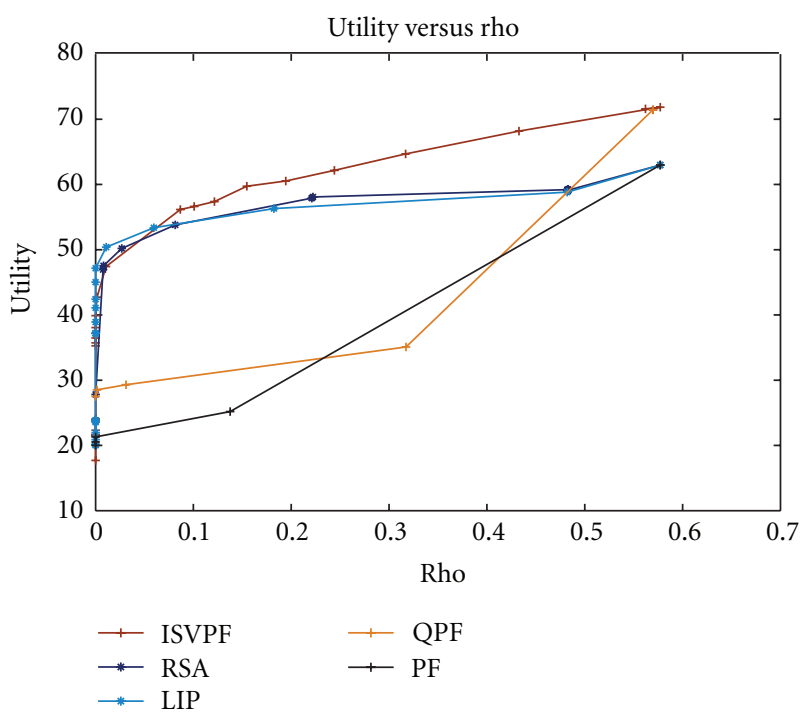

Figure 11: Utility $(\widehat{\Psi})$ versus Rho $\left(\widehat{\rho_{d}}\right)$ when queue length $=500$.

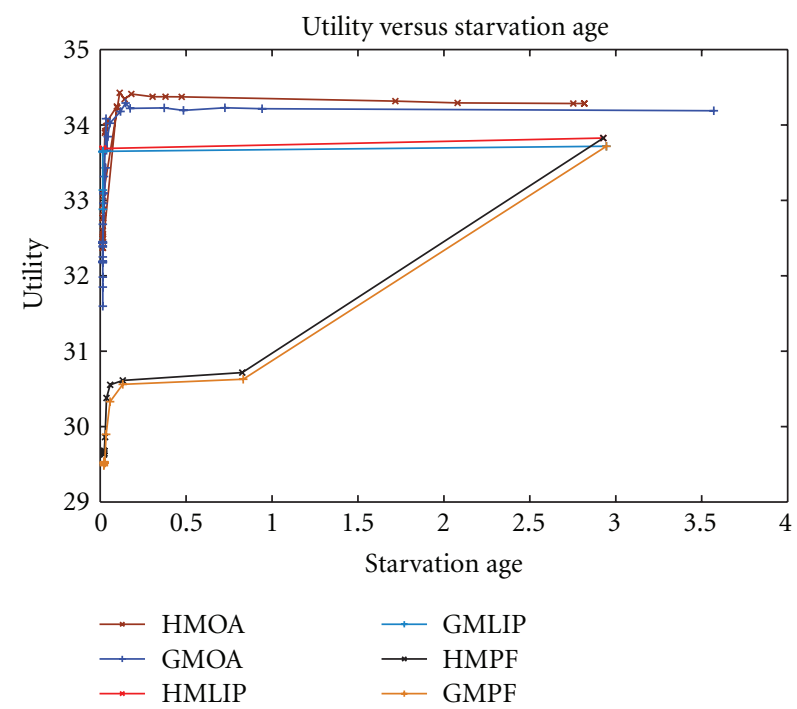

FIgURE 12: Utility $(\hat{\Psi})$ versus Starvation Age $(\hat{\chi})$.

Greedy MultiChannel Allocation were derived for multiuser multichannel video scheduling. Simulation results demonstrate that the proposed HMOA and GMOA schemes achieve a significantly higher video quality for a given starvation age compared to standard LIP and PF policies.

\section{Notation}

$U: \quad$ Number of users

$\mathcal{V}(m, n)$ : Video stream with $m$ temporal layers and $n$ quantization layers

$\mathcal{R}(m, n)$ : Bit rate of $\mathcal{V}(m, n)$

$\mathcal{Q}(m, n):$ Quality of $\mathcal{V}(m, n)$

$U(m, n)$ : Utility of video stream

$n: \quad$ Time slot index

$s^{n}: \quad$ State of the channel at slot $n$

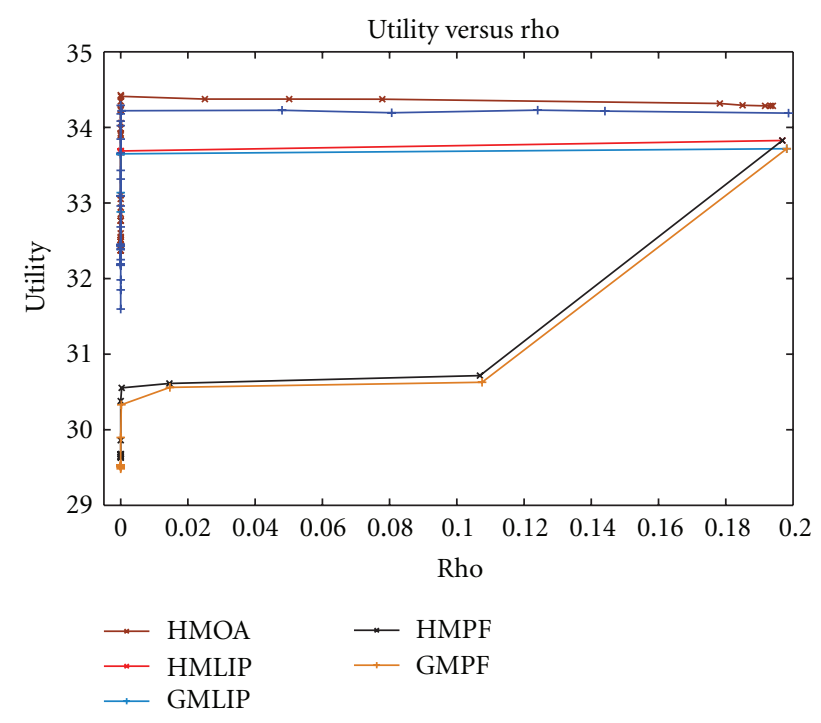

Figure 13: Utility $(\hat{\Psi})$ versus Rho $(\hat{\chi})$.

L: $\quad$ Number of channel states

$v^{n}$ : $\quad$ Video state of a HOL frame at slot $n$

$a^{n}: \quad$ Starvation age at slot $n$

$\mathcal{R}(s)$ : Maximum bit rate of channel at state $s$

$\mathbf{q}_{u}^{n}$ : $\quad$ Vector of utilities of packets of user $u$ at time slot $n$

$L_{u}: \quad$ Maximum queue size of user $u$

$\tau$ : $\quad$ Damping coefficient of PF policy

$\chi: \quad$ Expected starvation age

$\beta$ : $\quad$ Probability that a channel state remains in same state

$N$ : $\quad$ Number of channels

$\tilde{\mathcal{V}}(m, n)$ : Video stream of $m$ th temporal layer and $n$th quantization layer

$\widetilde{\mathcal{R}}(m, n)$ : Bit rate of $\tilde{\mathcal{V}}(m, n)$

$\widetilde{\mathcal{Q}}(m, n)$ : Quality of $\tilde{\mathcal{V}}(m, n)$

$\omega(n)$ : Set of users scheduled at time slot $n$

$u$ : User index

$\mathbf{s}^{n}$ : $\quad$ Joint channel state of all users at slot $n$

$\mathbf{P}^{u}$ : $\quad$ Probability transition matrix of user $u$

$\mathbf{v}^{n}$ : $\quad$ Joint video state of all users at slot $n$

$\mathbf{a}^{n}$ : $\quad$ Joint starvation age of all users at slot $n$

$K$ : Weight factor for starvation age

$\mathbf{q}^{n}$ : Joint vector of utilitiesvector of all users at

time slot $n$

$h_{r}: \quad$ Bias term of random policy $r$

$\Psi: \quad \quad$ Expected per slot long-term utility

$\rho_{d}$ : Probability that user is not served for morethan $d$ slots

$P: \quad$ Number of sample paths

PF: $\quad$ Proportional Fair

ISVPF: Index based scalable video scheduling Policy for finite queue size

QoS: Quality of service

GOP: Group of pictures

JSVM: Joint Scalable Video Model 
QPF: Quality Proportional Fair policy

HMOA: Hungarian Multi-Channel Optimal Allocation

HMPF: Hungarian Matching Proportional Fair policy

GMPF: Greedy Matching Proportional Fair policy

MDP: Markov decision process

ISVP: Index based scalable video scheduling policy

SVC: $\quad$ Scalable video coding

HUF: Highest urgency first

QP: $\quad$ Quantization parameter

LIP: $\quad$ Linear Index Policy

RSA: Rate Starvation Age policy

HMLIP: Hungarian matching LIP

GMLIP: Greedy matching LIP

GMOA: Greedy Multichannel sub-Optimal Allocation.

\section{References}

[1] Y. N. Lin, Y. D. Lin, Y. C. Lai, and C. W. Wu, "Highest Urgency First (HUF): a latency and modulation aware bandwidth allocation algorithm for WiMAX base stations," Computer Communications, vol. 32, no. 2, pp. 332-342, 2009.

[2] K. Wongthavarawat and A. Ganz, "IEEE 802.16 based last mile broadband wireless military networks with quality of service support," in Proceedings of IEEE Military Communications Conference (MILCOM '03), vol. 2, pp. 779-784, October 2003.

[3] P. De Cuetos and K. W. Ross, "Optimal streaming of layered video: joint scheduling and error concealment," in Proceedings of the 11th ACM International Conference on Multimedia (MM '03), pp. 55-64, New York, NY, USA, November 2003.

[4] C. Chen, R. W. Heath Jr., A. C. Bovik, and G. De Veciana, "Adaptive policies for real-time video transmission: a Markov decision process framework," in Proceedings of the 18th IEEE International Conference on Image Processing (ICIP '11), pp. 2249-2252, Brussels, Belgium, September 2011.

[5] V. R. Reddyvari and A. K. Jagannatham, "Quality optimal policy for H.264 scalable video scheduling in broadband multimedia wireless networks," in Proceedings of the International Conference on Signal Processing and Communications (SPCOM '12), Indian Institute of Science, Bangalore, India, July 2012.

[6] D. Tse and P. Viswanath, Fundamentals of Wireless Communication, Cambridge University Press, New York, NY, USA, 2005.

[7] A. Goldsmith, Wireless Communications, Cambridge University Press, New York, NY, USA, 2005.

[8] X. Liu, E. K. P. Chong, and N. B. Shroff, "A framework for opportunistic scheduling in wireless networks," Computer Networks, vol. 41, no. 4, pp. 451-474, 2003.

[9] R. Agrawal, A. Bedekar, R. J. La, R. Pazhyannur, and V. Subramanian, "Class and channel condition based scheduler for EDGE/GPRS," in Modelling and Design of Wireless Networks, Proceeding of SPIE, pp. 59-69, August 2001.

[10] J. Kim, J. Cho, and H. Shin, "Resource allocation for scalable video broadcast in wireless cellular networks," in Proceedings of IEEE International Conference on Wireless and Mobile Computing, Networking and Communications (WiMob '05), pp. 174180, August 2005.

[11] "JSVM 9.19.14 (joint scalable video model) software for the scalable video coding (SVC) project of the joint video team (JVT) of the ISO/IEC moving pictures experts group (MPEG)".
[12] Y. Wang, Z. Ma, and Y. F. Ou, "Modeling rate and perceptual quality of scalable video as functions of quantization and frame rate and its application in scalable video adaptation," in Proceedings of the 17th International Packet Video Workshop (PV'09), pp. 1-9, May 2009.

[13] http://media.xiph.org/video/derf/.

[14] Y. F. Ou, T. Liu, Z. Zhao, Z. Ma, and Y. Wang, "Modeling the impact of frame rate on perceptual quality of video," in Proceedings of the IEEE International Conference on Image Processing (ICIP' 08), pp. 689-692, October 2008.

[15] N. Bolia and V. Kulkarni, "Index policies for resource allocation in wireless networks," IEEE Transactions on Vehicular Technology, vol. 58, no. 4, pp. 1823-1835, 2009.

[16] V. Kulakarni, Modeling and Analysis of Stochastic Systems, Chapman and Hall, New York, NY, USA, 1995.

[17] M. Puterman, Markov Decision Processes: Discrete Stochastic Dynamic Programming, John Wiley \& Sons, New York, NY, USA, 1994.

[18] P. Bender, P. Black, M. Grob, R. Padovani, N. Sindhushayana, and A. Viterbi, "CDMA/HDR: a bandwidth-efficient highspeed wireless data service for nomadic users," IEEE Communications Magazine, vol. 38, no. 7, pp. 70-77, 2000. 

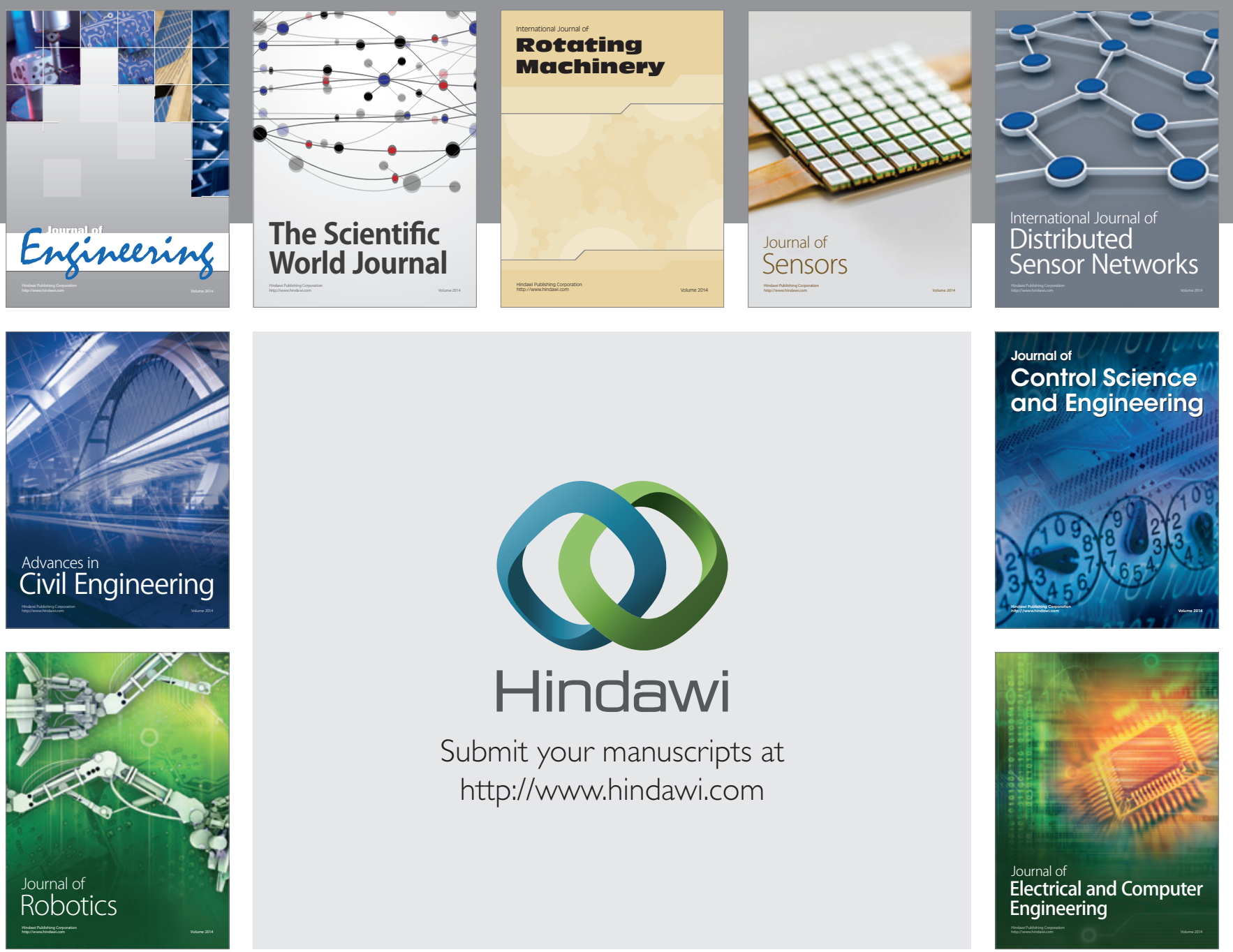

Submit your manuscripts at

http://www.hindawi.com
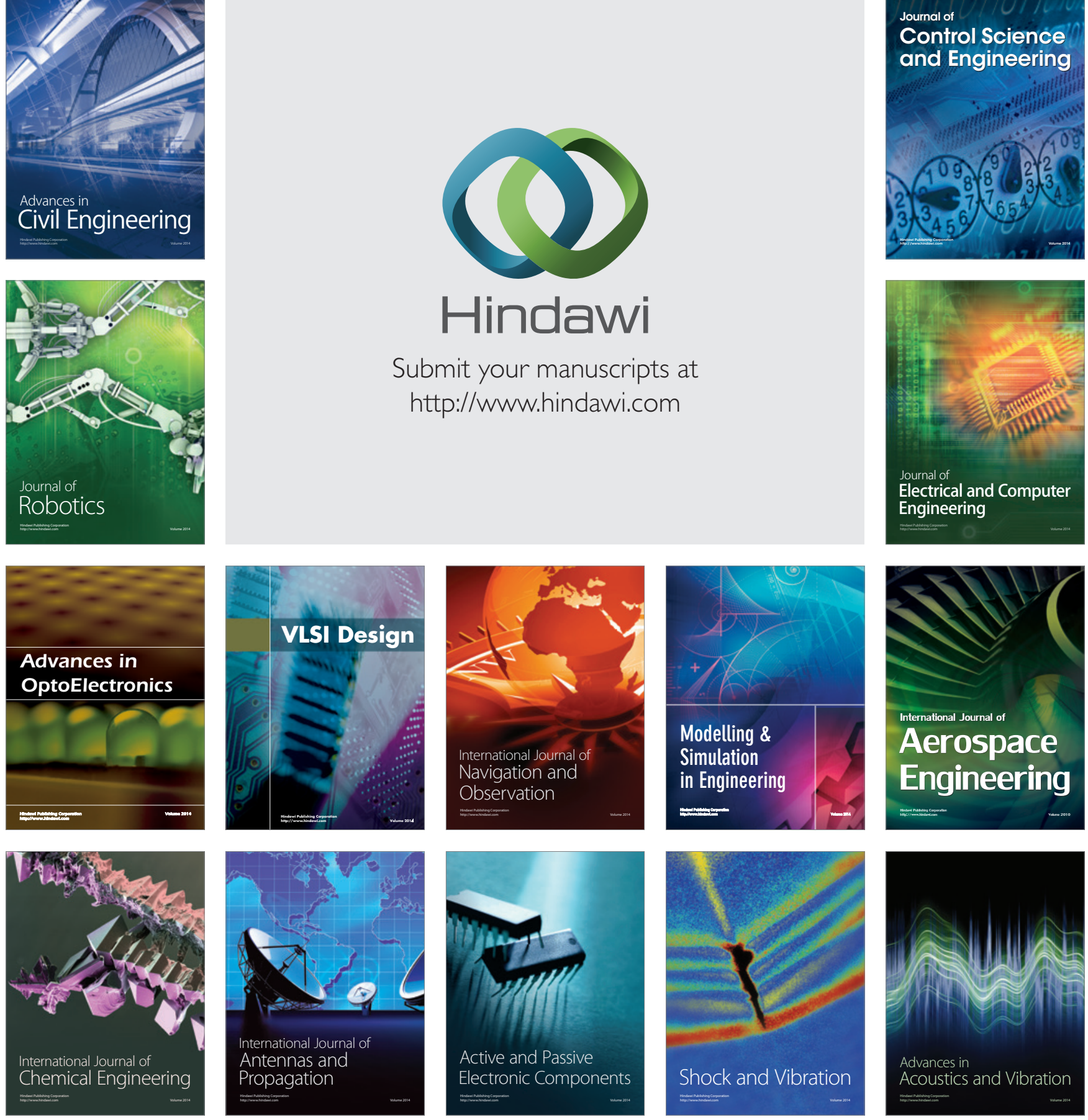